\title{
LAS ESTRATEGIAS DE APRENDIZAJE Y LAS RELACIONES FAMILIARES EN ESTUDIANTES DE SECUNDARIA
}

\author{
Jose L. Antoñanzas Laborda \\ Profesor de la Facultad de Educación, Universidad de Zaragoza \\ jlantona@unizar.es \\ Ángela Lope Álvarez \\ Profesora de la Facultad de Educación, Universidad de Zaragoza \\ Tania Carbonell Ventura \\ Master en la Universidad Oberta de Catalunya
}

Fecha de Recepción: 19 Febrero 2018

Fecha de Admisión: 10 Abril 2018

\section{RESUMEN}

Uno de los grandes retos de la educación del Siglo XXI es adaptarse a las distintas formas de aprender de los alumnos. En este sentido han sido varias las reformas educativas que se han realizado en los últimos años y todas ellas, obviamente, hacen referencia a los aprendizajes de los estudiantes. Sin lugar a dudas, las últimas tecnologías, y por ende la repercusión que tiene en los jóvenes, están cambiando no sólo las formas de aprender si no también las maneras de relacionarse. Son muchos los estudios que se han ido realizando sobre como aprenden los alumnos, a lo largo del tiempo. Pero son menos frecuentes las investigaciones que se centran en las relaciones familiares y los hábitos de estudio y estrategias de aprendizaje que estos utilizan.

Por todo ello se creó esta investigación, que pretende conocer un poco mejor aspectos fundamentales de los jóvenes en la educación secundaria como son sus hábitos y lugares de estudio, sus estrategias de aprendizaje y también como perciben sus relaciones familiares.

Palabras clave: estrategias de aprendizaje; técnicas de estudio; estudiantes; familia

\section{ABSTRACT}

Learning strategies and family relationships in high school students.

One of the great challenges of education in the 21 st century is to adapt to the different ways of learning from the students. In this sense there have been several educational reforms that have been made in recent years and all of them obviously refer to the student's learning. Undoubtedly, the latest technologies, and therefore the impact on young people, are changing not only the ways of learning but also the ways of relating. There are many studies that have been carried out on how students learn, over time. But the research Centrann in family relationships and the study habits and learning strategies they use are less FAQs. 


\section{LAS ESTRATEGIAS DE APRENDIZAJE Y LAS RELACIONES FAMILIARES EN ESTUDIANTES DE SECUNDARIA}

That is why this research was created, which aims to know a little better fundamental aspects of young people in secondary education such as their habits and places of study, their learning strategies and also how they perceive their family relationships.

Keywords: learning strategies; study techniques; students; family

\section{ESTRATEGIAS DE APRENDIZAJE Y HÁBITOS DE ESTUDIO EN SECUNDARIA}

A lo largo del tiempo las estrategias de aprendizaje se han venido definiendo como los pensamientos y comportamientos que sirven para adquirir y comprender mejor los nuevos conocimientos y habilidades (Ingles, Martinez y Garcia, 2013; Weinstein, Husman y Dierking, 2000). Ya en 1986 Weinstein y Mayer definían las estrategias de aprendizaje como aquellos conocimientos que un estudiante utiliza para aprender y que influye en su proceso de codificación, de tal forma que mejora la adquisición y la recuperación de lo aprendido. Para estos autores existen cuatro tipos estrategias: De repetición, de elaboración, de organización y estrategias afectivas. Por otra parte y desde el procesamiento de la información y posiciones mas constructivistas sobre el aprendizaje (Ausubel, 1983; Beltran, 1996; Gagné, 1965; Feuerstein, Rand y Hofmann, 1980; Flavell, 1977; Sternberg, 1986) se infiere que en el aprendizaje existen una serie de procesos cognitivos u operaciones mentales organizadas y coordinadas a partir de la conducta del sujeto, cuando este se tiene que enfrentar a tareas de razonamiento o resolución de problemas, y dichos procesos funcionan como las metas a alcanzar por las estrategias de aprendizaje que utiliza dicho sujeto. Dentro de esta concepción las estrategias de aprendizaje son actividades propositivas y que tienen su reflejo en las cuatro grandes fases del procesamiento de la información.

No cabe duda que el saber estudiar con eficacia y hacer un buen uso de la metodología de estudio es algo básico e imprescindible para el alumno y a su vez una constante preocupación, tanto par docentes como para toda la comunidad educativa. A lo largo del tiempo y según diverso autores (Beltran, 2003; Monereo, 2003; Pozo, 1990) lo mas importante en el proceso de enseñanza aprendizaje es dotar al alumnado de instrumentos y herramientas para que aprenda significativamente, es decir, que "aprenda a aprender". En este sentido las técnicas de estudio son útiles y necesarias para ello, ya que permiten gestionar y procesar la información que debe alcanzarse estableciendo un puente entre esa nueva información y la que ya se conoce; pero por sí solas no garantizan el dominio de las estrategias de estudio. El propio sistema educativo debe de favorecer y posibilitar, dentro del proceso enseñanza-aprendizaje, cuándo, cómo y porqué utilizar una determinada técnica. A su vez, desarrolla en los estudiantes estrategias de aprendizaje, con el propósito que ellos puedan convertirse en aprendices reflexivos y puedan adquirir de esta forma, los conocimientos que están siempre en constante transformación en la sociedad (Zumalacárregui, Mondeja 2007). Autores como Pozo, Monereo, Castelló, (2001) reivindican la necesidad de que las estrategias de aprendizaje se enseñen al mismo tiempo que se enseñan los contenidos pertenecientes a cada disciplina. Para Beltrán (2003) las estrategias de aprendizaje son las grandes herramientas del pensamiento, ya que son de gran utilidad tanto para potenciar como para extender su acción en cualquier lugar y momento donde se emplean. Asi como Las herramientas físicas potencian la acción física del hombre, las herramientas mentales potencian la acción del pensamiento.

Por otra parte, parece fundamental también hacer referencia a los hábitos de estudio. El concepto de hábito de estudio hace referencia al modo de afrontar las tareas académicas por parte del alumno. Es decir son las costumbres que tiene el individuo de organizar su espacio, su tiempo, sus técnicas a la hora de estudiar (Belaunde,1994).

Existen una gran cantidad de estudios que reconocen una asociación entre hábitos de estudio y rendimiento académico (véase De Tagle, Osornio, Heshiki y Garcés, 2008; Sánchez, Flores y Flores 
2016; Sánchez Briseño, 2016 y Paredes Ayrac, 2008). Para autores como Rondón (1991), tienen una importancia significativa en el mejoramiento del rendimiento académico de los estudiantes. Lo mismo opinan Aduna y Marquez (1987), quienes concluyen que los hábitos de estudio son una buena base para que los alumnos tengan mejores resultados.

También hay que destacar a la familia, como parte importante en el proceso de desarrollo del sujeto y su influencia en el rendimiento académico del mismo. Sin lugar a dudas, la familia es uno de los bases de influencia, a través de la cual el individuo desarrollará su personalidad, sus conductas, aprendizajes y valores. Para Musitu y García, (2001) tiene una función socializadora, ya que el individuo acepta y aplica las normas que esta le impone. Para otros autores (Grusec, 2002; Palacios, 1999b), las influencias familiares son las primeras y las más persistentes, ya que se caracterizan por ser especialmente intensas a nivel afectivo y tienen un gran peso sobre las relaciones posteriores fuera de la familia. En relación con el rendimiento académico, Hess y Holloway (1984) identificaron cinco procesos que vinculan a la familia y al desempeño académico. Desde el intercambio verbal, pasando por las expectativas familiares sobre el propio rendimiento académico, las relaciones positivas dentro de la familia, las creencias de los padres acerca de sus hijos, las atribuciones que estos hacen de su comportamiento hasta las estrategias de control y disciplina. Mas recientemente Vallejo y Mazadiego (2006), proponen estudiar los estilos parentales en relación con el rendimiento académico. Para dichos autores no solo existe ya evidencia sólida de la forma en que los padres influyen en el rendimiento académico de las hijas e hijos desde la infancia hasta la adultez. También se cuenta con estrategias metodológicas que han demostrado ser útiles para la investigación sobre este tópico que van desde estudios

observacionales hasta el uso extensivo de cuestionarios en estudios de tipo transversal o longitudinal. En particular, el rendimiento académico ha sido abordado con diversas estrategias para evaluarlo que van desde las calificaciones hasta la trayectoria y expectativas educativas de los estudiantes, encontrándose que la influencia de los estilos parentales en el desempeño académico se presenta desde la infancia hasta la adultez.

\section{METODOLOGÍA}

Sujetos. - la muestra está compuesta por alumnos de la Eso y de Bachillerato (2016/17) de un Instituto Público de la ciudad de Zaragoza. En total han sido 419 cuestionarios los recopilados.

Instrumentos.- Se adaptó el cuestionario de Hábitos de estudio y de estrategias de aprendizaje de la Universidad de Granada para alumnos de educación secundaria. Los apartados del mismo son los siguientes:

Datos sociodemográficos del alumno y de los padres.

Hábitos de estudio y lugar de estudio.

Planificación del tiempo y del estudio.

Toma de apuntes.

Estrategias de aprendizaje.

Afrontamiento de exámenes.

Satisfacción.

Y por otra parte se uso la prueba ESFA (Barraca y Lopez, 2010), para medir la satisfacción con la familia por parte del sujeto. Este cuestionario consta de 27 items y trata de evocar una respuesta de tipo afectivo preferentemente. Su finalidad es la evaluación de la satisfacción familiar expresada por los sujetos a través de distintos adjetivos. El tiempo requerido es de unos diez minutos.

Procedimiento.- El pase de pruebas se realizó por investigadores de la Universidad de Zaragoza y profesores /tutores del IES Pignatelli de los distintos cursos. El periodo de pase de pruebas fue de cuatro semanas . 


\section{LAS ESTRATEGIAS DE APRENDIZAJE Y LAS RELACIONES FAMILIARES EN ESTUDIANTES DE SECUNDARIA}

Una vez recopilados los datos, estos fueron introducidos en el programa estadístico SPSS 21. Los resultados que se presentan a continuación corresponden a los análisis de frecuencias de los distintos ítems que comprenden el cuestionario de hábitos de estudio y de estrategias de aprendizaje de la Universidad de Granada.

El número de alumnos que realizaron los cuestionarios fue de $\mathrm{N}=525,106$ de los cuales resul$\tan$ no validos. Por lo tanto, se realizará este informe teniendo en cuenta a 419 expedientes registrados. De la dicha muestra, 215 son varones $(51,3 \%)$ y 204 son mujeres $(48,7 \%)$ (Ver Fig,1). En relación con la edad de los estudiantes, y según se refleja en la tabla I, las edades comprendían desde los 12 hasta los 19 años, siendo la franja de 13 a 15 años la que más porcentaje representan respecto de la muestra general $(64 \%)$.

Fig.1. Distribución por sexo

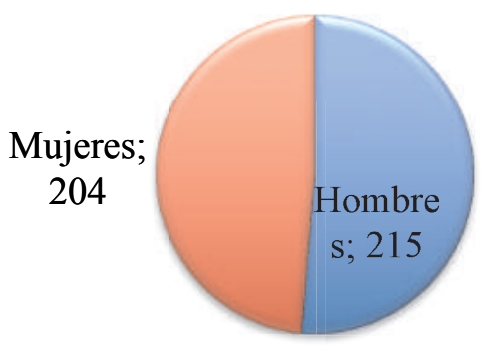

Tabla 1. Edad (2015/2016)

\begin{tabular}{|ccc|}
\hline Edad & Frecuencia & Porcentaje \\
\hline 12 años & 48 & 11,5 \\
\hline 13 años & 88 & 21 \\
\hline 14 años & 87 & 20,8 \\
\hline 16 años & 93 & 22,2 \\
\hline 17 años & 45 & 10,7 \\
\hline 18 años & 41 & 9,8 \\
\hline 19 años & 13 & 3,1 \\
\hline
\end{tabular}

Por otra parte, y según se aprecia en la Fig.2, el mayor número de alumnos se encuentran en 1ํㅡำ de la ESO, siendo el número de estudiantes de bachillerato el menos numeroso.

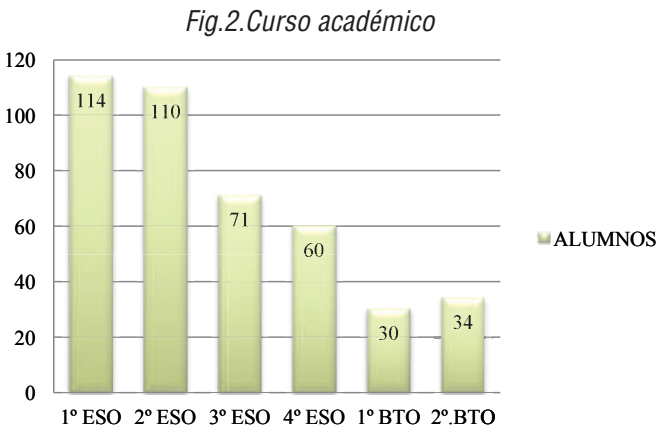


En relación con quién viven los alumnos (Tabla 2), la mayoría lo hace con los padres ( $\mathrm{N}=326)$ lo que representa un $77,8 \%$ de la muestra. Existe un número significativo de alumnos que lo hace con la madre ( $N=64 ; 15,1 \%)$. Además, cuando se les pregunta a los alumnos con quién pasan más tiempo, el porcentaje que contesta con su madre se eleva a un $45,8 \%$ y solo un $8,6 \%$ lo hace con el padre, el resto lo hace con los dos por igual, 44,6\%. En cuanto a la relación de los padres, el $69,9 \%$ de los padres están casados, frente a un $22,4 \%$ que están separados y un $5,7 \%$ viven en pareja sin estar casados.

Tabla 2. Con quién vives

\begin{tabular}{|ccc|}
\hline & Frecuencia & Porcentaje \\
\hline Padres & 326 & 77,8 \\
\hline Padre & 15 & 3,6 \\
\hline Madre & 64 & 15,3 \\
\hline Abuelos & 2 & 0,5 \\
\hline Otros & 12 & 2,9 \\
\hline
\end{tabular}

En cuanto al nivel académico de los padres (Tabla 3), se refleja que el $46,6 \%$ de las madres terminaron sus estudios básicos, tanto EGB o la ESO, y de los padres un 53\%. Coinciden en porcentaje, los padres y madres que terminaron el bachillerato (17,4\%). Encontramos diferencias entre las madres que terminaron una carrera universitaria con los padres (madres: $23,4 \%$, padres: $16 \%$ ).

Tabla 3. Estudios padres (2015/2016)

\begin{tabular}{|ccc|}
\hline & Nivel madre & Nivel padre \\
\hline Ns/Nc & $13,1 \%$ & $13,6 \%$ \\
\hline EGB & $28,4 \%$ & $35,1 \%$ \\
\hline ESO & $17,7 \%$ & $17,9 \%$ \\
\hline Bachillerato & $17,4 \%$ & $17,4 \%$ \\
\hline Universidad & $23,4 \%$ & $16 \%$ \\
\hline
\end{tabular}

\section{Hábitos de estudio y lugar del diseño.}

Los alumnos han respondido acerca de la percepción que tienen los padres sobre ellos y, el $70,4 \%$ de ellos han respondido que sus padres le consideran buen estudiante, mientras que el $26,5 \%$ estiman lo contrario (ver Fig 3.).

A la hora de realizar los deberes, el $30,5 \%$ afirma que recibe ayuda, el $68 \%$ de los alumnos asegura no lo contrario. Sin embargo, cuando se les pregunta quién les ayuda un $50,8 \%$ contesta que nadie, un $15,5 \%$ de los alumnos afirman son academias o profesores particulares quienes cubren esta labor. Un $4,1 \%$ dice que es el padre quién ayuda y el porcentaje aumenta a un $8,1 \%$ al tratarse de la madre. Al $11,8 \%$ de los alumnos le ayudan su padre y su madre en partes iguales. 
Fig, 3. Ayuda en los deberes

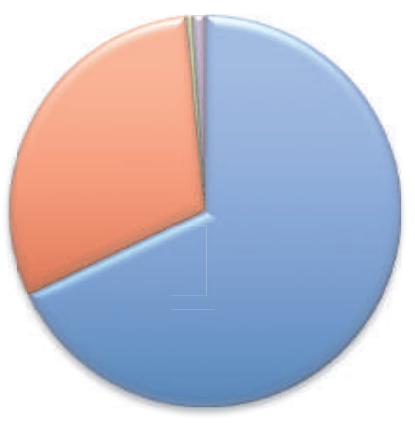

$\square$ Sin ayuda

Con ayuda

$\checkmark$ A veces

$\square \mathrm{Ns} / \mathrm{nc}$

Tabla 4 Ayuda recibida en los deberes (2015/2016)

\begin{tabular}{|ccc|}
\hline & Frecuencia & Porcentaje \\
\hline Nadie & 212 & $50,6 \%$ \\
Padre & 17 & $4,1 \%$ \\
Madre & 34 & $8,1 \%$ \\
\hline Madre y hermanos & 1 & $0,2 \%$ \\
Madre y Padre & 49 & $11,7 \%$ \\
\hline Padres y hermanos & 3 & $0,7 \%$ \\
Hermanos & 33 & $7,9 \%$ \\
Otros & 65 & $15,5 \%$
\end{tabular}

Una cuestión importante a la hora de estudiar, es el lugar en que los alumnos lo hacen habitualmente (Fig. 4). Se les preguntó si disponían de un lugar fijo para el estudio. El 91,9\% manifestó que si que tenía un lugar fijo para estudiar. De ellos, un 83,9\% lo hace en su habitación y un 9,3\% en el salón. El $64 \%$ de los alumnos afirman que no comparten el lugar de estudio con ningún otro miembro de la familia frente a un $26,7 \%$ que dice que lo comparte a veces. Los que comparten lo hacen habitualmente con hermanos (25,7\%). Por otra parte, y en relación con el lugar de estudio, un $58,9 \%$ de los alumnos disponen de ordenador, además disponen de luz natural un $79,5 \%$. En cuanto a la temperatura, un $29,6 \%$ responde que no tiene una temperatura agradable a la hora de estudiar y que un $43,7 \%$ no tiene silencio en su lugar de estudio. Con todo ello, un $83,3 \%$ considera su lugar de estudio idóneo.

Fig. 4 Disponen de...

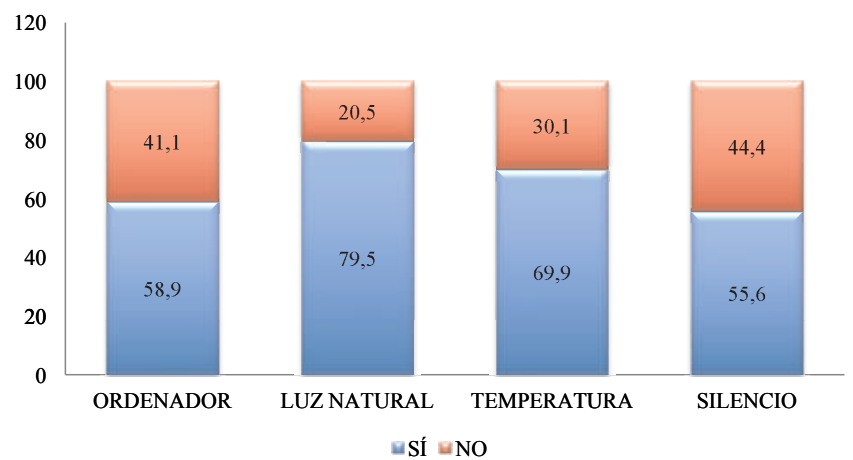




\section{Planificación y distribución del tiempo}

Se les preguntó acerca de cómo es el momento del estudio y las respuestas han sido las siguientes. Un $25,1 \%$ de los alumnos dice consigue estudiar sin distracción desde un 75 a $100 \%$ del tiempo que invierten estudiando. Es significativo que más de la mitad de los alumnos (53,7\%) afirmen que consiguen estudiar entre 0 y50\% del tiempo sin distracciones. El $87,2 \%$ de los alumnos asegura que no suele estudiar viendo la televisión (de un 0-25\% del tiempo que estudian) y tampoco suelen escuchar música $71,3 \%$. También es significativo el porcentaje de tiempo que estudian recostados, un $37,9 \%$ dice no hacerlo nunca, pero de 0-25\% del tiempo hay un 70,4\% que sí lo hace.

En general, cuando se les pregunta sobre la planificación del tiempo, un 48,4\% de los alumnos afirman que a veces se planifican el tiempo, un $23,4 \%$ dice planificarlo siempre frente a un $15,5 \%$ que nunca lo planifica (ver Fig, 5). Un 59,7\% asegura que cumple con la planificación frente a un $36 \%$ que no lo cumple. En relación con la planificación de contenidos, un 22,9\% lo planifica siempre y un $11 \%$ nunca. Un $76 \%$ de los alumnos cumple con la planificación de contenidos. Se les preguntó también si confeccionaban un calendario de estudio en el que indicaran los días y las horas que deben estudiar. Solo un 5,3\% dio una respuesta afirmativa, un 59,7\% no lo crea nunca. En cuanto a su cumplimiento, el $49,6 \%$ no lo cumple y un $31,7 \%$ sí.

Fig. 5, Planificación del tiempo dedicado al estudio

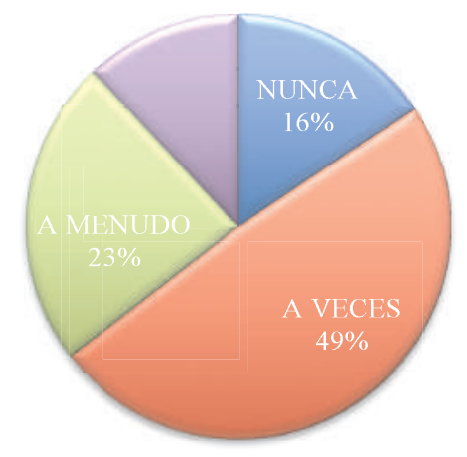

Una cuestión importante es saber cuándo estudian, un 24,1\% dice comenzar con el inicio del curso, un $73 \%$ sólo cuando se acercan los exámenes. En cuanto a qué días suelen estudiar, un $43 \%$ estudia todos los días, un 18,4\% solo estudia los fines de semana. En relación con el tiempo dedicado al estudio, solo un $7,9 \%$ prepara todas las asignaturas por igual, mientras que un $88,3 \%$ las estudia en función de la fecha de examen. Un 57,3\% comienza a estudiar por las asignaturas más fáciles. Y, durante el estudio, un $62,5 \%$ afirma levantarse del sitio, solo un 7,6\% no se levanta nunca. El $21,5 \%$ de los alumnos afirman que a menudo están cansados en el momento del estudio.

\section{Toma de apuntes}

A continuación se presentan los resultados de cómo toman apuntes los alumnos. Destaca que un $36,5 \%$ de los alumnos dice no tomar apuntes. También existe un $74,9 \%$ de estudiantes que copia al pie de la letra lo que dice el profesor. Llama la atención que un poco menos de la mitad de lo alumnos no tenga la iniciativa de preguntar al profesor cuando tiene una duda. Se eleva mucho el porcentaje al preguntar a los alumnos si fotocopian los apuntes de sus compañeros, la mayoría contesta que no. Se acerca a la igualdad el porcentaje cuando les preguntamos si se sienten cómodos realizando los trabajos de forma individual o en grupo (Ver Tabla 5). 


\section{LAS ESTRATEGIAS DE APRENDIZAJE Y LAS RELACIONES FAMILIARES EN ESTUDIANTES DE SECUNDARIA}

Tabla 5. Modo en que toma apuntes el alumno (2016/2017)

\begin{tabular}{|c|c|c|c|}
\hline & Sí & NO & $\mathrm{Ns} / \mathrm{Ne}$ \\
\hline Tomo apuntes de las explicaciones del profesor/a & $63,5 \%$ & $34,4 \%$ & $2,1 \%$ \\
\hline $\begin{array}{l}\text { Cuando tomo apuntes copio al pie de la letra lo que dice el } \\
\text { profesor/a }\end{array}$ & $23,2 \% \%$ & $74,9 \%$ & $1,9 \%$ \\
\hline $\begin{array}{l}\text { Tengo dificultades para seguir las explicaciones del profesor/a en } \\
\text { clase }\end{array}$ & $19,1 \%$ & $79 \%$ & $1,9 \%$ \\
\hline Cuando tengo dudas pregunto al profesor/a & $56,1 \%$ & $42 \%$ & $1,9 \%$ \\
\hline Fotocopio los apuntes de algún compañero/a & $9,3 \%$ & $88,8 \%$ & $1,9 \%$ \\
\hline Me siento más cómodo realizando los trabajos de forma individual & $42,2 \%$ & $55,8 \%$ & 1,9 \\
\hline
\end{tabular}

\section{Estrategias de aprendizaje}

En relación a las estrategias que emplean los estudiantes a la hora de estudiar cabe destacar las siguientes: Los alumnos no relacionan contenidos de una asignatura con otras $(79,7 \%)$. Tampoco amplían información con bibliografía complementaria (89,5\%). La mayoría de ellos tampoco utiliza mapas conceptuales de los temas para estudiar $(87,8 \%)$. También aseguran que no consultan los temas que no entienden en otras fuentes de información $(74,2 \%)$.

Por otra parte, muchos de ellos sí subrayan el material de estudio $(71,1 \%)$, realizan resumen de Ios temas a estudiar $(65,2 \%)$, memorizan los apunte para el día del examen $(66,1 \%)$ y se plantean preguntas en el momento del estudio que podrían salir en el examen (69,9\%). Otro aspecto a valorar es la utilización de muchas palabras en la realización de esquemas, un 87,8\% asegura no utilizarlas (Ver Tabla 6).

Tabla 6. Estrategias de estudio (2015/2016)

\begin{tabular}{|c|c|c|c|}
\hline & SI & NO & $\mathrm{Ns} / \mathrm{Ne}$ \\
\hline $\begin{array}{l}\text { Cuando estudio relaciono los contenidos de la materia con otras } \\
\text { asignaturas }\end{array}$ & $18,4 \%$ & $79,7 \%$ & $1,9 \%$ \\
\hline Amplío información con bibliografía complementaria & $8,6 \%$ & $89,5 \%$ & $1,9 \%$ \\
\hline $\begin{array}{l}\text { Cuando estudio dispongo de toda información y materiales } \\
\text { necesarios }\end{array}$ & $60,9 \%$ & $37,2 \%$ & $1,9 \%$ \\
\hline $\begin{array}{l}\text { Antes de estudiar en profundidad suelo hacer una lectura } \\
\text { superficial }\end{array}$ & $55,8 \%$ & $42,2 \%$ & $1,9 \%$ \\
\hline Subrayo el material de estudio & $71,1 \%$ & $27 \%$ & $1,9 \%$ \\
\hline Hago esquemas del material a estudiar & $48,4 \%$ & $49,6 \%$ & $1,9 \%$ \\
\hline En la realización de esquemas utilizo muchas palabras & $10,3 \%$ & $87,8 \%$ & $1,9 \%$ \\
\hline Realizo un resumen de los temas a estudiar & $65,2 \%$ & $32,9 \%$ & $1,9 \%$ \\
\hline $\begin{array}{l}\text { Hago mapas conceptuales de los temas de cada una de las materias } \\
\text { para estudiar posteriormente }\end{array}$ & $10,3 \%$ & $87,8 \%$ & $1,9 \%$ \\
\hline $\begin{array}{l}\text { Los temas que no entiendo los consulto en un diccionario, } \\
\text { enciclopedia,... }\end{array}$ & $23,9 \%$ & $74,2 \%$ & $1,9 \%$ \\
\hline Memorizo los apuntes para el día del examen & $66,1 \%$ & $32 \%$ & $1,9 \%$ \\
\hline $\begin{array}{l}\text { Cambio mi forma de estudiar si el examen es de test o de } \\
\text { desarrollo }\end{array}$ & $36,8 \%$ & $61,3 \%$ & $1,9 \%$ \\
\hline $\begin{array}{l}\text { Cuando estudio para un examen me planteo preguntas que pueden } \\
\text { incluirse en el examen. }\end{array}$ & $69,9 \%$ & $28,2 \%$ & $1,9 \%$ \\
\hline
\end{tabular}

\section{Modo con el que afrontan los exámenes}

En relación a como afrontan los exámenes los alumnos, el 73,5\% de los alumnos se dedica a repasar el día anterior al examen y también la mayoría lo repasan antes de entregarlo al profesor $(70,4 \%)$. También afirman que muchos de ellos suelen dormir poco la noche anterior al examen $(73,3 \%)$ y que comienzan por la primer pregunta sin leer el resto $(71,6 \%)$ (Ver Tabla 7$)$. 
Tabla 7. Modo de afrontar los exámenes (2015/2016)

\begin{tabular}{|c|c|c|c|}
\hline & Sí & NO & $\mathrm{Ns} / \mathrm{Ne}$ \\
\hline El día anterior al examen lo dedico a repasar & $73,5 \%$ & $24,6 \%$ & $1,9 \%$ \\
\hline La noche anterior al examen suelo dormir poco & $24,8 \%$ & $73,3 \%$ & $1,9 \%$ \\
\hline Cuando tengo un examen me pongo nervioso/a & $56,6 \%$ & $41,5 \%$ & $1,9 \%$ \\
\hline $\begin{array}{l}\text { Cuando realizo un examen comienzo por la primera pregunta sin } \\
\text { leer el resto }\end{array}$ & $25,5 \%$ & $71,6 \%$ & $1,9 \%$ \\
\hline Repaso el examen antes de entregarlo al profesor & $70,4 \%$ & $27,7 \%$ & $1,9 \%$ \\
\hline $\begin{array}{l}\text { Al salir del examen compruebo con los apuntes la respuesta que } \\
\text { he dado }\end{array}$ & $54,4 \%$ & $43,7 \%$ & $1,9 \%$ \\
\hline Suelo recordar lo estudiado después del examen & $40,8 \%$ & $57,3 \%$ & $1,9 \%$ \\
\hline
\end{tabular}

\section{Satisfacción}

Se les preguntó a los alumnos si se sentían satisfechos con las calificaciones que obtuvieron el curso anterior. Un $57 \%$ contestaron que sí lo estaban, frente a un $39.9 \%$ que dio una respuesta negativa (Fig.6).

Fig. 6 Satisfacción con las calificaciones del curso anterior

$$
\square \text { Sí } \square \mathrm{NO} \bullet \mathrm{Ns} / \mathrm{Nc}
$$

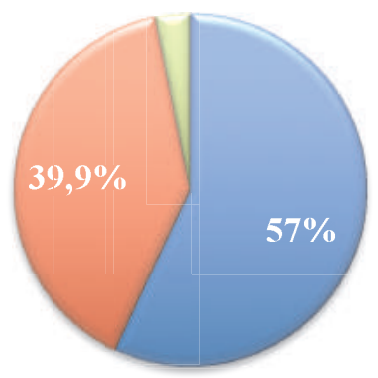

En relación con la satisfacción familiar las diferencias también son significativas (ver tabla 8). En este caso son los mas jóvenes, de 12 a 15 años, los que tienen mayor índice de satisfacción familiar (33\% y 22,80\%) o si se observa por abajo, son los jóvenes de 16 a 19 años los que dicen tener menos satisfacción en sus relaciones familiares (83,90\% y 73,30\%).

Tabla 8. Satisfacción con la familia.

\begin{tabular}{llllllll} 
& Edad & $12-13$ & $14-15$ & $16-17$ & $18-19$ & & \\
\hline $\begin{array}{l}\text { Satisfacción } \\
\text { familiar }\end{array}$ & MUY ALTO & $11,00 \%$ & $11,40 \%$ & $0,00 \%$ & $13,30 \%$ &, 040 & $17,244^{*}$ \\
\hline & & & & & & & \\
& ALTO & $22,00 \%$ & $11,40 \%$ & $16,10 \%$ & $13,30 \%$ & & \\
& NORMAL & $36,00 \%$ & $29,50 \%$ & $61,30 \%$ & $33,30 \%$ & & \\
& BAJO & $31,00 \%$ & $47,70 \%$ & $22,60 \%$ & $40,00 \%$ & & \\
\hline
\end{tabular}

\section{CONCLUSIONES.}

Durante el año académico 2016/2017, los cuestionarios fueron pasados a 419 alumnos de los cuales aunque la franja de edad estaba comprendida entre los 12 a19 años, la franja que más porcentaje ha sido representado han sido alumnos entre los 13 y 15 años respecto a la muestra general siendo estos los alumnos de la ESO. 


\section{LAS ESTRATEGIAS DE APRENDIZAJE Y LAS RELACIONES FAMILIARES EN ESTUDIANTES DE SECUNDARIA}

Partiendo de los datos anteriores, encontramos que la mayoría de los alumnos viven con los padres en el mismo núcleo, frente a una minoría significativa que lo hacen con la madre. En relación con quien pasan más tiempo, la mayoría pasan más tiempo con la madre que con la minoría que presenta el padre, pero también vemos que casi se elevan las cifras de los dos padres frente a la de la madre, es decir, estadísticamente el porcentaje de los que pasan más tiempo con la madre 0 con ambos padres a la vez es el mismo.

Respecto al nivel académico de los padres es de la EGB o la ESO. Si tenemos en cuenta la percepción que tienen los padres sobre los alumnos, vemos que el $70 \%$ de los encuestados los consideran buenos estudiantes mientras que el porcentaje restante estiman lo contrario, que son malos estudiantes. En este caso sobre la ayuda que reciben los alumnos llama la atención respecto a otros cursos anteriores que no precisan de ayuda académica, pero los que resultantes de la ayuda respecto a otros años afirman que son academias o profesores particulares y no sus padres quienes cubren dicha labor.

Los estudiantes cuando estudian es preciso que tengan un lugar fijo de estudio y más del $90 \%$ de estudiantes admiten que lo tienen, el resto de alumnos lo comparten con algún miembro de la familia o lo comparte algunas veces. Es notable la importancia que le dan los alumnos a tener un ordenador presente en el lugar de estudio, además de disponer una luz natural 0 una temperatura agradable. Respecto a la planificación del estudio, la mayoría de los encuestados invierten bien el tiempo estudiando y consiguen hacerlos sin distracciones así mismo el estudio depende del tiempo y para ello es imprescindible tener una buena organización. Los estudiantes que tienen una buena organización tienden a planificarse y para ello utilizan un calendario de estudio en el que indican las horas y los días que deben estudiar. En relación a los encuestados, tres cuartas partes de los mismos no saben coger apuntes ya que copian todo lo que dice el profesor al pie de la letra sin destacar unas frases de otros, mientras que un tercio de los encuestados no toman ningún tipo de apuntes. También es interesante destacar que los alumnos no saben relacionar contenidos de una asignatura con las de otras, tampoco amplían la información dada en clase con bibliografía complementaria, no utilizan mapas conceptuales.

En relación con la satisfacción con la familia, en general los jóvenes parecen tener unas buenas relaciones con la misma. Ahora bien, también conforme cumplen mas años la satisfacción con la familia disminuye, existe lo que se denomina conflictos familiares. aunque en general dicho nivel de insatisfacción es bajo. Datos que concuerdan con estudios anteriores donde se percibía un bajo conflicto entre adolescentes y familia (Rodrigo, García, Márquez y Triana, 2005). La satisfacción con las relaciones familiares de los jóvenes se vera mermada conforme estos van creciendo, aunque si bien dicha relación se puede considerar como buena.

\section{REFERENCIAS}

Aduna, M., \& Marquez, S. (1987). Curso de hábitos de estudio y autocontrol. México: Trillas S.A.

Ausubel, D (1983). Teoría del Aprendizaje Significativo. Consultado en: http://www.monografías. com/trabajos6/ apsi/apsi.shtml.

Beltrán, J. (1996). Procesos, estrategias y técnicas de aprendizaje. Madrid: Síntesis Psicología.

Palacios, J. (1999b): La familia y su papel en el desarrollo afectivo y social. En F. lópez, I. Etxebarría, M.J. Fuentes y M.J. Ortiz (eds.), Desarrollo afectivo y social (267-284) Madrid

Beltrán, J. (2003). Estrategias de aprendizaje. Revista de Educación, 332, 55-73.

Chilca, M.L. (2017). Autoestima, hábitos de estudio y rendimiento académico en estudiantes universitarios. Propósitos y representaciones, 5(1) 71-127.

De Tagle, R., Osornio, L., Heshiki, N. y Garcés, L. (2008). Hábitos de estudio y rendimiento escolar 
en alumnos regulares e irregulares de la carrera de médico cirujano de la FESI, UNAM. En: Revista Electrónica de Psicología Iztacala, Universidad Nacional Autónoma de México, 11(2), 117.

Feuerstein, R.; Rand, Y. y Hoffman, M.D. (1980) Instrumental enrichemen. An intervention program for cognitive modificability. Baltimore: University Press.

Flavell, J.H. (1977) El desarrollo cognitivo. Madrid: VISOR

Gagne. R. (1965). The corulitions of learning. New York Holt, Rjnchart \& Winston.

Grusec, J.E. (2002): Parental socialization and children s acquisition of values. En M.H. BORNSTEIN (Ed.), Handbook of Parenting. Vol 5. Practical issuesin parenting;143-167. Mahwah, N.J., Erlbaum.

Hess, R. D. y Holloway, S. D. (1984) "Family and school as educational institutions". In R. D. Parke(Ed), review of child development research VII, 179-222). Chicago:

University of Chicago Press.

Inglés,C.J., Martínez,A.E.y Garcia, J.M.(2013). Conducta prosocial y estrategias de aprendizaje en una muestra de estudiantes españoles de Educación Secundaria Obligatoria. European Journal of Education and Psychology. 6,(1), 33-53.

Monereo, C. (coord.) (2000). Las estrategias de aprendizaje. Madrid: Visor.

Musitu, G. y García, J. F. (2001). Escala de socialización parental en la adolescencia (ESPA 29). Madrid: TEA.

Paredes Ayrac, D. M. (2008). Relación entre la satisfacción con la profesión elegida, los hábitos de estudio y el rendimiento académico de los estudiantes de la UNSAM (Tesis inédita). Universidad Nacional Mayor de San Marcos, Lima.

Pozo, J.I. (1990). Estrategias de aprendizaje. En C. Coll,J. Palacios y A. Marchesi (Eds.), Desarrollo psicológico y educación, II. Psicología de la Educación (pp.199-221). Madrid: Alianza.

Pozo, J.I., Monereo, C. y Castelló, M. (2001) El uso estratégico del conocimiento. En Coll. C., Palacios, J. y A. Marchesi (comp.) Desarrollo psicológico y educación, 2. Psicología de la educación escolar. Madrid: Alianza (pp. 211-234).

Rodrigo, Mạ.J., García, M., Márquez, M.L. y Triana, B. (2005) Discrepancias entre padres e hijos adolescentes en la frecuencia percibida e intensidad emocional en los conflictos familiares. Estudios de Psicología, 26 (1): 21-34.

Rondón, C. (1991). Internalidad y Hábitos de Estudio. Carabobo: Universidad Pedagógica Experimental Libertador.

Sánchez R., Flores, N., \& Flores. F. (2016). Influencia de los hábitos de estudio en el rendimiento académico de los estudiantes de una institución de educación media ecuatoriana. LatinAmerican Journal of Physics Education, 10(1), 1-7.

Sanchez Briceño, S. (2016). Relación entre hábitos de estudio y rendimiento académico en estudiantes del tercer año de secundaria de una institución educativa del distrito de San Martin de Porres, provincia de Lima, año 2015 (Tesis de Licenciatura). Universidad Alas Peruanas, Lima. Recuperado de http://repositorio.uap.edu.pe/handle/uap/2278

Sternberg, R. J. (1986). A triangular theory of love. Psychological Review, 93(2), 119-135.

Vallejo Casarín, A., \& Mazadiego Infante, T.J. (2006). Familia y rendimiento académico. Revista de Educación y Desarrollo, 5, 55-59.

Weinstein, C.E., Husman, J. y Dierking, D.R. (2000). Self-regulation interventions with a focus on learning strategies. En M. Boekaerts, P. R. Pintrich, y M. Zeidner (Eds.), Handbook of self-regulation (pp. 728-748). San Diego, CA: Academic Press.

Weinstein, C. and Mayer, R. (1986) The Teaching of Learning Strategies. In: Wittrock, M., Ed., 
Handbook of Research on Teaching, Macmillan, New York, 315-327.

Zumalacárregui, Mondeja (2007). Los Enfoques Ciencia, Tecnología Y Sociedad Como Recurso Didáctico En La Química Universitaria. Revista Pedagogía Universitaria, XII(5). 